\title{
Eubacterium alactolyticum
}

National Cancer Institute

\section{Source}

National Cancer Institute. Eubacterium alactolyticum. NCI Thesaurus. Code C86384.

A genus of aerobic, Gram-positive, rod shaped bacteria assigned to the phylum

Firmicutes. This species is nonmotile, non-spore forming, indole negative, reduces

neutral red, but not nitrate, does not produce hydrogen sulfide, and does not hydrolyze hippurate, esculin, or starch. E. alactolyticum, also known as Pseudoramibacter alactolyticus, has been isolated from dental calculus and ging ival crevices in individuals with periodontal disease. 